\title{
Identification of seven novel variants in the $\beta$-globin gene in transfusion-dependent and normal patients
}

\author{
Sumayh A. Aldakeel ${ }^{1}$, Neda Z. Ghanem ${ }^{1}$, Amani M. Al-Amodi ${ }^{1}$, Ahoud Khalid Osman'1, \\ Lubna Ibrahim Al Asoom², Nazish Rafique Ahmed², Noor B. Almandil'3, Mohammed Shakil Akhtar', \\ Sayed Abdul Azeez ${ }^{1}$, J. Francis Borgio ${ }^{1}$
}

\begin{abstract}
${ }^{1}$ Department of Genetic Research, Institute for Research and Medical Consultation (IRMC), Imam Abdulrahman Bin Faisal University, Dammam, Saudi Arabia 2Department of Physiology, College of Medicine, Imam Abdulrahman Bin Faisal University, Dammam, Saudi Arabia

${ }^{3}$ Department of Clinical Pharmacy Research, Institute for Research and Medical Consultation (IRMC), Imam Abdulrahman Bin Faisal University, Dammam, Saudi Arabia

${ }^{4}$ Department of Biochemistry, College of Medicine, Imam Abdulrahman Bin Faisal University, Dammam, Saudi Arabia
\end{abstract}

Submitted: 6 April 2018

Accepted: 20 September 2018

Arch Med Sci 2020; 16 (2): 453-459

DOI: https://doi.org/10.5114/aoms.2019.84825

Copyright $\odot 2019$ Termedia \& Banach

\section{Abstract}

Introduction: Abnormality in $H B B$ results in an inherited recessive blood disorder, which can be caused by variants at the transcriptional or translational level affecting the stability and the production of the HBB chain. The severity of the disease relies on the variant's characteristics. This study aimed to identify the common $\beta$-globin $H B B$ variants in the population of the Eastern Province, which has the highest prevalence of blood diseases in Saudi Arabia. Material and methods: Direct sequence of $\beta$-globin $H B B$ gene, and alpha-globin HBA1 and HBA2 genes was performed on a total of 545 blood samples (transfusion-dependent: 215, 106 men and 109 women; normal healthy subjects: 330, 197 men and 133 women) collected from Saudi Arabian participants in the Eastern region.

Results: A total of 36 variants in $H B B$ gene were revealed with 11 variants that have been reported for the first time in Saudi Arabia, including 7 novel variants that have been identified for the first time in $H B B$ gene. The novel variants consisted of two exonic (HBB:c.252C>T; HBB:c.281G>T) and five intronic variants (c.316-183 316-168del; c. $315+241 \mathrm{~T}>\mathrm{A} ; \quad$ c. $315+376 \mathrm{~T}>\mathrm{C}$; c.316-114C>G; c.315+208T>G) at $H B B$ gene. The novel exonic variants and three (c.316-183 316-168del; c.315+241T>A; c.315+376T>C) intronic variants were co-inherited with $\alpha$ deletion.

Conclusions: This current study updated the $H B B$ gene variations with newly identified variants of $H B B$ gene and co-inheritance with $\alpha$-globin deletions. The identified $\beta$-globin mutations will strengthen the genetic reference that could aid in characterizing mutations that are associated with phenotype of thalassemia in a specific region.

Key words: $H B B$ gene, novel variants, hemoglobin, hematological disorders, DNA sequencing.

\section{Introduction}

$\beta$-Globin protein (HBB), one of the hemoglobin subunits, is produced by $\beta$-globin gene $(H B B)$, which is located on chromosome 11 [1]. Two $\beta$-globin

\author{
Corresponding author: \\ Dr. J. Francis Borgio \\ Department \\ of Genetic Research \\ Institute for Research and \\ Medical Consultation (IRMC) \\ Imam Abdulrahman Bin \\ Faisal University \\ Dammam, Saudi Arabia \\ Phone: + 966133330864 \\ E-mail: fbalexander@iau.edu.sa; \\ borgiomicro@gmail.com
}


molecules bind to two $\alpha$-globin molecules to constitute the most popular form of hemoglobin, adult hemoglobin $(\mathrm{HbA})$. HBB is crucial in balancing the ratio of $(\alpha: \beta)$-globin chains, preventing the aggregation of insoluble $\alpha$-globin complex [1-3]. Abnormality in the $H B B$ gene results in an inherited recessive blood disorder that can be caused by variants at the transcription or translation level affecting the stability and the production of the $\beta$-globin chain $[4,5]$.

Several $H B B$ variants are produced by mutations in $H B B$ gene, and some mutations alters the production of HBB chain either partially $\left(\beta^{+}\right)$or completely $\left(\beta^{\circ}\right)$ [6]. Reduced formation of HBB chain lowers the amount of functional $\mathrm{Hb}$, which is a characteristic of the highly prevalent blood disorder in Saudi Arabia, $\beta$-thalassemia [7-12]. Variations in HBB protein can also be associated with other genetic hematological disorders such as sickle cell disease, which is very common in Saudi Arabia. To date, there are more than 1,700 hemoglobin variants that have been reported, with more than 900 variants in $H B B$ [13]; however, the most common variants are hemoglobin $\mathrm{E}(\mathrm{HbE})$, sickle hemoglobin $(\mathrm{HbS})$, and hemoglobin $\mathrm{C}(\mathrm{HbC})$ that results as a consequence of point mutations in $H B B$ gene [14]. The formation of the highly unstable $\alpha$ and $\beta$ complex $\mathrm{HbE}$ is a result of a point mutation at position 26 in $H B B$ that substituted glutamic acid with lysine, causing a phenotypic characteristic of a mild form of $\beta$-thalassemia [15]. During the production of HbS, the most common cause of sickle cell disease is a consequence of a point mutation at the sixth position in $H B B$ gene that substituted glutamic acid codon (GAG) with valine codon (GTG) [16]. Additionally, glutamic acid can be substituted with lysine forming $\mathrm{HbC}$, which is a $\mathrm{Hb}$ variant that is related to sickle cell disease [5]. On the other hand, some variants might have beneficial influences. For instance, it has been known that individuals with $\mathrm{HbC}$ are protected at different level against Plasmodium falciparum that cause malaria $[17,18]$.

The severity of the disease relies on some factors such as the variant's characteristics and the imbalance ratio of $\alpha: \beta$ chains [19]. There are about $42 \mathrm{HBB}$ variants that have been identified in the population of Saudi Arabia [7]. These mutations either reduce $\left(\beta^{+}\right)$or prevent $\left(\beta^{\circ}\right)$ the synthesis of HBB protein. Therefore, identification of $H B B$ genes variants will be helpful to understand the heterogeneity and prevalence of pathogenic variants in the population of Eastern Province. Thus, this study was conducted in an effort to identify the $H B B$ gene variants in the population of the Eastern Province of Saudi Arabia.

\section{Material and methods}

\section{Samples collection}

A total of 545 blood samples were collected from male $(n=303)$ and female $(n=242)$ par- ticipants from the Eastern region of Saudi Arabia. This study included 215 transfusion-dependent subjects (age range: 2 months to 51 years; 106 males and 109 females) and 330 normal healthy subjects (age range: 8 months to 67 years; 197 males and 133 females), who attended major hospitals in the region. Samples were requested from random volunteers and patients who were clinically diagnosed with $\beta / \alpha$-thalassemia major/ carriers or sickle cell anemia. Transfusion-dependent subjects include 20 sickle cell disease (homozygous) patients and $195 \beta$-thalassemia major patients. Subjects with no history of blood transfusion, blood disorders, or chronic diseases were included as normal control.

The study was approved by the Standing Committee for Research Ethics on Living Creatures, Imam Abdulrahman Bin Faisal University (CBME2012032; IRB-2015-08-069).

\section{Hematological parameters}

Hematological parameters were evaluated following the collection of blood samples $(5 \mathrm{ml})$ in EDTA-coated vacutainers using Coulter Micro Diff II (Beckman Coulter, Inc., Brea, CA, USA) and VARIANT $^{\text {TM }}$ II Hemoglobin Testing System (BIO-Rad Laboratories, Inc., Hercules, CA, USA).

\section{DNA extraction and sequencing}

DNA was extracted from all of the 545 blood samples utilizing QIAamp DNA blood minikit (Qiagen, $\mathrm{GmbH}$, Hilden, Germany) followed by amplification of $H B B, H B A 1$ and $H B A 2$ genes using the PCR standard method [8, 20-22]. Utilizing forward and reverse primers separately, $H B B, H B A 1$, and $H B A 2$ genes in each sample were amplified by PCR using BigDye Terminator Cycle Sequencing Kit (Thermo Fisher Scientific, Inc., Waltham, MA, USA). Following the amplification of each gene, purification was conducted to prepare the samples for sequencing, which was performed using the series Genetic Analyzer 3500 (Thermo Fisher Scientific, Inc.) at the Department of Genetic Research, Institute for Research and Medical Consultation, Imam Abdulrahman Bin Faisal University (Dammam, Saudi Arabia). DNA sequencing analysis software v. 5.3 (Applied Biosystem; Thermo Fisher Scientific, Inc.) and mutation surveyor software (Softgenetics, US) were used to analyze electropherograms.

\section{Results}

Conducting a direct sequence of HBB gene from 545 (215 transfusion-dependent subjects and 330 normal healthy subjects) subjects revealed that the 249 subjects were with normal $H B B$ gene and 296 subjects were identified 
with 36 (HBB:C.118C>T; HBB:C.315+1G>A; HBB: C.93-21 96del; HBB:C.20A>T; HBB:C.92+5G>C; HBB:c.92+6T>C; HBB:c.17-18delCT; HBB:c.9321G>A; HBB:c.25_26delAA; HBB:c.9T>C; HBB:c. 27_28insG; HBB:c.79G>A; HBB:c.92+1G>A; HBB: C.431T>A; HBB:C.2T>C; HBB:c.112delT; HBB:C.315 +74T>G; HBB:C.415G>A; HBB:C.432C >T; HBB:C. 435G >A; HBB:C.281G>T; HBB:C.26A>T; HBB:C.92+ 2T>C; HBB:c.218G>C; HBB:c.253A>G; HBB:c.294 C>T; HBB:C.135delC; HBB:C.93-1G>C; HBB:C.370 A>C; HBB:C.320T>C; HBB:C.315+208T>G; HBB:C. 315+241T>A; HBB:C.315+376T>C; HBB:C.316-183 316-168del; HBB:c.252C>T, and HBB:c.316-114 $\bar{C}>G$ ) different types ( $\beta^{\circ}$ : a complete lack of $\beta$-globin production; $\beta^{+}$: a partial production of $\beta$-globin; $\beta^{\mathrm{S}}$ : sickled; $\beta^{\text {i }}$ : intronic mutation; whereas $\beta^{\mathrm{E}}$ is $\mathrm{HbE}$ disease) of variants in the exonic and intronic regions of $H B B$ gene. Among the $36 H B B$ variants, 25 variants have been previously reported from the Saudi Arabian population, the remaining 11 variants have been identified for the first time in this study in Saudi Arabians. Among these 11 variants, 7 ( 2 exonic and 5 intronic) variants have been identified for the first time in $H B B$ gene in the study subjects. These 7 variants (HBB:c.281G>T; HBB:c.316-183_316-168del; HBB:C.315+208T>G; HBB:C.315+241T>A; HBB:C.315+376T>C; HBB:C. 252C>T; and HBB:c.316-114C>G) have not been reported earlier in any population; hence, these 7 variants were described in detail in Table I and are referred as novel variants.

Twenty-six $H B B$ variants (HBB:C.118C $>$ T; HBB:C. 20A>T; HBB:C.315+1G>A; HBB:c.93-21_96del; HBB: C.92+5G>C; HBB:C.93-21G>A; HBB:c.17-18delCT; HBB:c.25_26delAA; HBB:c.27_28insG; HBB:c.79G>A; HBB:c.431T>A; HBB:c.2T>C; HBB:c.112delT; HBB: c.315+74T>G; HBB:c.415G>A; HBB:c.432C>T; HBB: c.435G>A; HBB:C.281G>T; HBB:c.9T>C; HBB:c.92+ 2T>C; BB:C.218G>C; HBB:C.370A>C; HBB:C.315+ 208T>G; HBB:C.315+241T>A; HBB:C.315+376T>C; and HBB:c.316-114C>G) have been observed in 86 subjects included as normal control group. Out of the 86 subjects, 42 were observed with $\beta^{\text {s, }} 3$ were observed with $\beta^{\mathrm{E}}, 22$ were observed with $\beta^{0}, 3$ were observed with $\beta^{+}$, and 4 were observed with $\beta^{i}$. Twenty-two HBB variants (HBB:c.118C $>$ T; HBB: c.20A > T; HBB:c.315+1G>A; HBB:c.93-21 96del; HBB: C.92+5G>C; HBB:c.92+6T>C; HBB:c.93-21G>; HBB:c. 17-18delCT; HBB:C.25_26delAA; HBB:c.27_28insG; HBB:c.112delT; HBB:c.415G>A; HBB:c.281G>T; HBB: C.316-183_316-168del; HBB:c.92+1G>A; HBB:c. 26A>T; HBB:C.253A>G; HBB:C.294C>T; HBB:C.135 delC; HBB:c.93-1G>C; HBB:c.320T>C; and HBB:C.252 $C>T$ ) have been observed in the transfusion-dependent subjects. Where 20 subjects were observed with homozygous $\beta^{\text {s }}$, remaining transfusion-dependent subjects were observed with homozygous $\beta^{0}$, homozygous $\beta^{+}$, or with compound heterozygous $\beta^{0}$ and $\beta^{+}$.
Two males and 3 females transfusion-dependent subjects had a total of four novel variants; 3 point mutations; HBB:C.252C $>$ T, HBB:C.281G $>$ T, and HBB:c.316-114C $>\mathrm{G}$ as well as one deletion; HBB: c.316-183_316-168del (Figure 1, Table I). The HBB:C.252C $>T$ is a silent point mutation, where the change in the codon results on encoding for the same amino acid, glycine. The point mutation; HBB:c.281G>T is a missense mutation, which results on encoding for the amino acid phenylalanine rather than cysteine. The point mutation, HBB:c.316-114C >G is present in the intronic region of $H B B$ gene. The newly reported deletion (HBB:c.316-183 316-168del) was observed in two transfusion-dependent patients who are clinically diagnosed with sickle cell anemia. The most common $\alpha$-thalassemia mutation amongst these transfusion-dependent subjects was the $3.7 \mathrm{sin}$ gle gene deletion. However, one of the subjects had HBA1 intronic mutation (Table I).

On the other hand, four newly identified point mutations have been observed in normal control participants (2 males, 1 female): HBB:c.281G>T, HBB:c.315+208T>G, HBB:c.315+241T>A, and HBB:c.315+376T>C (Figure 1, Table I). HBB:c.281G>T is a point mutation that was identified also in a normal control subject, which results on the conversion of an UGU codon into UUU, which encodes the amino acid phenylalanine rather than cysteine. The remaining three point mutations: HBB:C. $315+208 T>G$, HBB:C. $315+241 T>A$, and HBB: C. $315+376 \mathrm{~T}>C$ are variants that present in the non-coding regions of $H B B$ gene (Figure 1 ). These novel $H B B$ variants in normal healthy group are co-inherited with $\alpha$-globin deletion.

Furthermore, in an effort to test the notion that the presence of these seven novel identified variants would have a more influence on red blood cell pathology compared to control/sickle cell/ thalassemia conditions, the general hematological parameters of blood samples were evaluated. In each sample, the co-inheritance of the novel mutation/mutations at the intronic regions seems to demonstrate no influence on blood parameters compared with existing of these conditions alone. However, one of the normal control subjects had a novel exonic missense mutation: HBB:c.281G $>T$ and borderline $\mathrm{HbA}_{2}$ level, i.e. 3\% (Table I).

\section{Discussion}

$H B B$ and $H B A$ genes encode the normal adult hemoglobin tetramer $(\mathrm{Hb})$, constituting of four polypeptide chains; $2 \alpha$ chains and $2 \beta$ chains [23]. Mutations such as frameshift, minor deletions, and missense mutations in the $H B B$ gene may result in highly unstable $\mathrm{HBB}$ protein $[6,24]$. Various novel mutations have been still observed in $H B B$ gene. Recently, Ekwattanakit et al. 


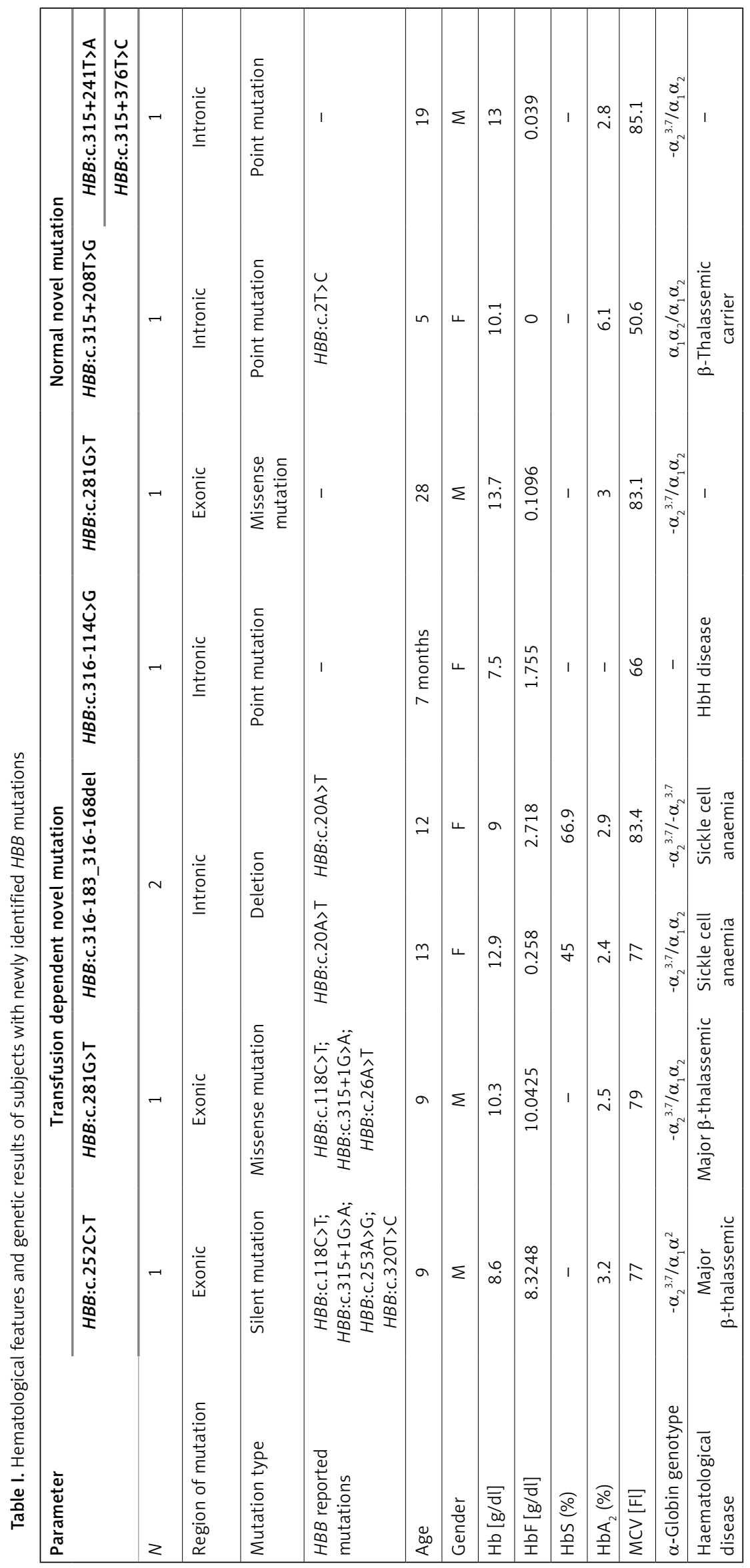




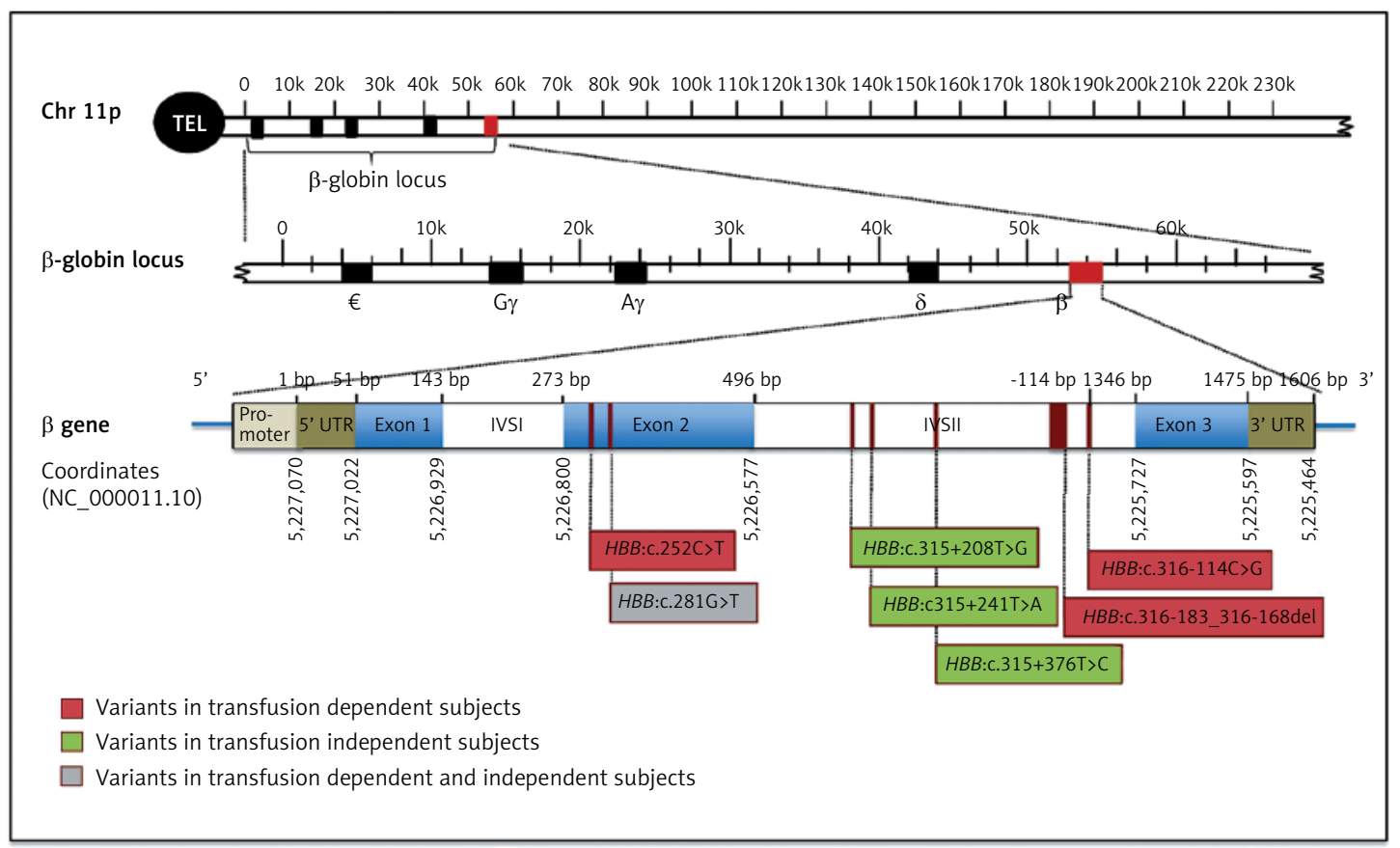

Figure 1. Schematic representation of HBB gene structure and novel identified variant locations. The position of the mutations is indicated below the gene. Novel mutations detected in subjects with transfusion-dependent are in red, mutations detected in normal subjects are in green, and the mutation identified in both subjects is in gray

[25] have identified two frameshift mutations in the third exon that causes a rapid mRNA decay in thalassemia intermedia patients. The novel heterozygous mutation identified in this present study, HBB:c.281G>T in exon 2 is caused by a missense mutation that substituted the sulfur containing amino acid, cysteine into the aromatic amino acid, phenylalanine [26]. This substitution might alter the protein structure or interfere with the heme-binding pocket as it has been shown that sulfur containing amino acids form stronger interactions compared to the aromatic amino acids. This substitution might reduce the stability of the protein function [26]. Further studies on these novel mutations are needed to investigate any alteration in the proper function of HBB protein.

$H B B$ produces an important component in building up hemoglobin complex, which carries on oxygen molecules into the cells. The production of $\beta$-globin chain affects the ratio of $\alpha$ and $\beta$, and in turns the production of an intact hemoglobin complex. In addition, the imbalance of $\alpha / \beta$ chains causes the formation of toxic complexes of free $\alpha$ chains aggregation $[2,27]$. There are several genetics hematological disorders associated with genetic changes in the $H B B$. This genetic change might yield unfunctional, structurally abnormal $\beta$-chain variants, or affect the proteins' production $[4,5]$. However, it has been previously found that the co-inheritance of $\beta$-mutations with $\alpha$-mutations might reduce the amount of $\alpha$-globin production, which in turn reduce the imbalance of $\beta / \alpha$ chain resulting in the amelioration of disease severity [28].

It is worth to note that these novel mutations that have been identified in this study are co-inherited with other mutations, which are present in $H B B$ gene or other genes that might directly interact with HBB. Therefore, it cannot be concluded whether the changes in the hematological parameters are influenced by the novel identified variants or as a result of other factors. To illustrate, the novel hetero-exonic mutation HBB:c.281G>T, which has one amino acid change from cysteine to phenylalanine, has been found solely in a $\beta$-thalassemia carrier as well as in a $\beta$-thalassemia major with other mutations. The $\beta$-thalassemia carrier subject had a borderline $\mathrm{HbA}_{2}$ level 3\% (Table I), which could be a consequence of either the mutation or the co-inheritance of $\alpha$ deletion $[3,7]$ as it has been previously shown to increase $\mathrm{HbA}_{2}$ level $[27,29]$. The severity of the phenotype in the $\beta$-thalassemia major subject might be affected by the presence of other mutations.

In an effort to understand the implications of these novel identified variants, number of future studies needs to be conducted. HBB gene consists of three exons and based on previous studies, the severity of the disease lies on which exon the mutations are located. For example, based on Cao and Galanello [6], the mutation in phase termination codon stimulates the nonsense-mediated mRNA decay (NMD) process, resulting 
in low production of normal HBB chain $[6,24]$. Since the two exonic novel mutations presented in this study are located in exon 2, further studies are required to investigate whether they have similar effects. Similarly, majority of the identified mutations in this study were present in intronic region, which can alter the gene's function and expression in different ways, as non-coding regions play a role in transcriptional and translational regulation. Non-coding mutations may also introduce novel splice sites that result in a truncated protein, leading in loss of function [30]. In addition, the effect of homozygosity of these mutations is not distinguished. Thus, future studies could overcome this limitation by investigating the effect of inheriting two mutant alleles of these novel variants on the severity of the phenotype.

In conclusion, $H B B$ molecular scanning for both transfusion-dependent and normal control subjects is an important step for better understanding and accurate diagnosis of hemoglobinopathies. This current study has identified 11 variants that have been reported for the first time in Saudi Arabia. Seven novel (2 exonic and 5 intronic) variants in $H B B$ gene have been observed in the study population. The influence of these novel variants on the phenotypic characteristics and $\beta$-globin proper function is currently unknown and thus needs to be examined. The identified $\beta$-globin mutations could be used as a genetic to the study region.

\section{Acknowledgments}

The authors would like to extend their appreciation to the Dean of the Institute for Research and Medical Consultation for the support and continuous encouragement. The study was supported by the Deanship of Scientific Research, Imam Abdulrahman Bin Faisal University (Grant No: 2012186; 2014024 and 2014051, 2015292; 2016-077-IRMC), the King Abdulaziz City for Science and Technology (Grant No. LGP-35-204; LGP32-3 and LGP-36-132), and the National Science Technology and Innovation Plan (Grant No: 12MED-2798-46). The authors would like to extend their appreciation to Mr. Ranilo M. Tumbaga, Mr. Horace T. Pacifico, and Ms. Jee E. Aquino for their technical support.

\section{Conflict of interest}

The authors declare no conflict of interest.

\section{References}

1. Morton CC, Kirsch IR, Taub R, Orkin SH, Brown JA. Localization of the beta-globin gene by chromosomal in situ hybridization. Am J Hum Genet 1984; 36: 576-85.

2. Khandros E, Thom CS, D'Souza J, Weiss MJ. Integrated protein quality-control pathways regulate free alpha- globin in murin beta-thalassemia. Blood 2012; 119: 5265-75.

3. Weatherall DJ, Clegg JB. Inherited haemoglobin disorders: an increasing global health problem. Bull World Health Organization 2001; 79: 704-12.

4. Yi P, Yu F, Huang S, et al. Identification of a novel frameshift mutation at codon $53(-\mathrm{T})$ in the beta-globin gene causing dominantly inherited beta-thalassemia in a Chinese Miao family. Blood Cells Mol Dis 2008; 41: 56-9.

5. Das SK, Talukder G. Beta globin gene and related diseases: a review. Int J Hum Genet 2002; 2: 139-52.

6. Cao A, Galanello R. Beta-thalassemia. Genet Med 2010; 12: 61-76.

7. Alaithan MA, AbdulAzeez S, Borgio JF. A comprehensive review of the prevalence of beta globin gene variations and the co-inheritance of related gene variants in Saudi Arabians with beta-thalassemia. Saudi Med J 2018; 39: 329-35.

8. Borgio JF, AbdulAzeez S, Al-Nafie AN, et al. A novel HBA2 gene conversion in cis or trans: " $\alpha 12$ allele" in a Saudi population. Blood Cells Mol Dis 2014; 53: 199-203.

9. Borgio JF. Molecular nature of alpha-globin genes in the Saudi population. Saudi Med J 2015; 36: 1271-6.

10. Borgio JF, Al-Madan MS, AbdulAzeez S. Mutation near the binding interfaces at alpha-hemoglobin stabilizing protein is highly pathogenic. Am J Transl Res 2016; 8: 4224-32.

11. Al-Nafie AN, Borgio JF, AbdulAzeez S, et al. Co-inheritance of novel ATRX gene mutation and globin (alpha and beta) gene mutations in transfusion dependent beta-thalassemia patients. Blood Cells Mol Dis 2015; 55: 27-9.

12. Akhtar MS, Qaw F, Borgio JF, et al. Spectrum o alpha-thalassemia mutations in transfusion-dependent betathalassemia patients from the Eastern Province of Saudi Arabia. Hemoglobin 2013; 37: 65-73.

13. Patrinos GP, Giardine B, Riemer C, et al. Improvements in the HbVar database of human hemoglobin variants and thalassemia mutations for population and sequence variation studies. Nucleic Acids Res 2004; 32 (Suppl 1): D537-541.

14. Wild BJ, Green BN, Cooper EK, et al. Rapid identification of hemoglobin variants by electrospray ionization mass spectrometry. Blood Cells Mol Dis 2001; 27: 691-704.

15. Fucharoen S, Weatherall DJ. The hemoglobin $E$ thalassemias. Cold Spring Harb Perspect Med 2012; 2: a011734.

16. Sun N, Zhao H. Seamless correction of the sickle cell disease mutation of the HBB gene in human induced pluripotent stem cells using TALENs. Biotechnol Bioeng 2014; 111: 1048-53.

17. Allison AC. Protection afforded by sickle-cell trait against subtertian malarial infection. Br Med J 1954; 1: 290-4.

18. Williams TN, Mwangi TW, Roberts DJ, et al. An immune basis for malaria protection by the sickle cell trait. PLoS Med 2005; 2: e128.

19. Origa R. Beta-thalassemia. Gen Med 2017; 19: 609-19.

20. Al-Amodi AM, Ghanem NZ, Aldakeel SA, et al. Hemoglobin $A_{2}\left(\mathrm{HbA}_{2}\right)$ has a measure of unreliability in diagnosing beta-thalassemia trait ( $\beta$-TT). Curr Med Res Opin 2018; 34: 945-51.

21. Borgio JF, AbdulAzeez S, Naserullah ZA, et al. Mutations in the beta-globin gene from a Saudi population: an update. Int J Lab Hematol 2016; 38: e38-40.

22. Memish ZA, Saeedi MY. Six-year outcome of the national premarital screening and genetic counseling program for sickle cell disease an beta-thalassemia in Saudi Arabia. Ann Saudi Med 2011; 31: 229-35. 
23. Rafiei J, Yavari K, Moosavi-Movahedi AA. Preferential role of iron in heme degradation of hemoglobin upon gamma irradiation. Int J Biol Macromolecules 2017; 103: 1087-95.

24. Thein SL. Is it dominantly inherited $\beta$ thalassaemia or just a $\beta$ thchain variant that is highly unstable? $\mathrm{Br} J$ Haematol 1999; 107: 12-21.

25. Ekwattanakit S, Riolueang S, Viprakasit V. Interaction between $\mathrm{Hb}$ E and $\mathrm{Hb}$ Yala (HBB:c. 129delT); a novel frameshift beta globin gene mutation, resulting in hemoglobin $E / \beta^{\circ}$ thalassemia. Hematology 2018; 23: 117-21.

26. Gómez-Tamayo JC, Cordomí A, Olivella M, Mayol E, Fourmy D, Pardo L. Analysis of the interactions of sulfur-containing amino acids in membrane proteins: interactions involving Met and Cys in membrane proteins. Protein Science 2016; 25: 1517-24.

27. Borgio JF, Abdulazeez S, Almandil NB, et al. The alpha3.7 deletion in alpha-globin genes increases the concentration of fetal hemoglobin and hemoglobin A2 in a Saudi Arabian population. Mol Med Reports 2018; 17: 1879-84.

28. Yavarian M, Karimi M, Bakker E, Harteveld C, Giordano P. Response to hydroxyurea treatment in Iranian transfusion-dependent beta-thalassemia patients. Haematologica 2004; 89: 1172-8.

29. Borgio JF, AbdulAzeez S, Al-Muslami AM, et al. KLF1 gene and borderline hemoglobin $A_{2}$ in Saudi population. Arch Med Sci 2018; 14: 230-6.

30. Faustino NA, Cooper TA. Pre-mRNA splicing and human disease. Gen Develop 2003; 17: 419-37. 